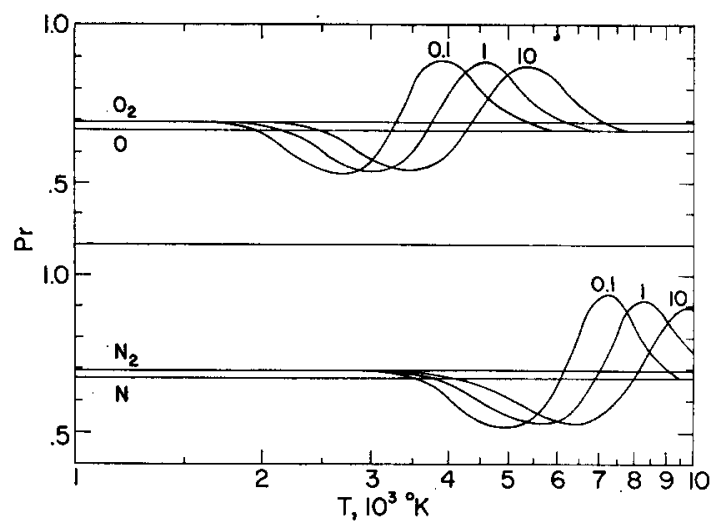

Fra. 5. Prandtl number of equilibrium dissociating nitrogen and oxygen at pressures of $0.1,1.0$, and $10 \mathrm{~atm}$.

viscosity of high-temperature oxygen has recently been measured by Hartunian and Marrone, ${ }^{22}$ and their results are shown in Fig. 2 for comparison with the calculations. The agreement is rather poor, a fact for which we have no ready explanation. Similar viscosity measurements have not been made for nitrogen, but its thermal conductivity has been measured up to $13000^{\circ} \mathrm{K}$ by Burhorn ${ }^{23}$ at 1 atm pressure by an arc technique. His experimental points are shown in Fig. 3. The abrupt rise at the highest temperatures is attributed to the onset of

${ }^{22}$ R. A. Hartunian and P. V. Marrone, Phys. Fluids 4, 535 (1961).

${ }^{23}$ F. Burhorn, Z. Physik 155, 42 (1959). ionization. When allowance is made for the probable ionization effects at high temperatures, the agreement seems satisfactory.

Also of experimental interest, although no measurements are yet available, is the Prandtl number,

$$
\operatorname{Pr}=\eta C_{p} / \lambda \bar{M},
$$

where $\bar{M}=x_{1} M_{1}+x_{2} M_{2}$ is the average molecular weight. The Prandtl number is shown in Fig. 5 for the same three pressures. Both $C_{p}$ and $\lambda$ have large maxima due to the chemical reaction, but they occur at slightly different temperatures and the result is an S-shaped curve. ${ }^{24}$

On the whole, no special unusual behavior seems to occur for nitrogen and oxygen, as it did for hydrogen. ${ }^{7}$

\section{ACKNOWLEDGMENTS}

The authors wish to thank R. T. Chen for his help with many of the calculations, and also Professor S. F. Singer and Colonel L. J. Poudre of the Physics Department for their kindness in making computation time available on their electronic computer.

This research was supported in part by the National Aeronautirs and Space Administration.

${ }^{24}$ C. F. Hansen, NACA TN 4150 (1958).

\title{
Microwave Probing of Ionized-Gas Flows
}

\author{
ROBERT G. JAHN* \\ Daniel and Florence Guggenheim Jet Propulsion Center, California Institute of Technology, Pasadena, California
}

(Received January 29, 1962)

\begin{abstract}
The measurement of free-electron densities and collision frequencies in ionized-gas flows by a probing transverse microwave beam is described. Interpretation of the observed reflection and transmission coefficients and their phases is developed on the basis of an idealized plane wave-plane slab model, for which typical results are displayed. Application of the device and technique to a specific experiment-the buildup of ionization behind a strong shock in argon-is outlined. Effects of various departures of the microwave radiation patterns and flow configurations from the slab assumptions are discussed and examples evaluated.
\end{abstract}

\section{INTRODUCTION}

$\mathbf{I}^{\mathrm{N}}$ $\mathrm{N}$ much of the current high-temperature gasdynamic research, profitable use could be made

* Now at Princeton University, Department of Aeronautical Engineering, The James Forrestal, Research Center. of a device which would measure the ionization density of a gas flow with the same convenience and reliability with which the pitot tube and interferometer measure the more familiar properties. In principle, there are several possible techniques for 
such ionization measurements: electrostatic probes, magnetic-induction probes, microwave interactions, spectroscopic line broadening and electron continuum techniques, and optical interferometry, but the development of any of these into a tool for routine aerodynamic measurement so far has been incomplete. Each is applicable only over a limited range of ionization density, and each has certain characteristic ambiguities in its response to an ionized-gas flow. Lacking any "standard source" of well-known, uniform ion density with which to calibrate them, such techniques as are applied to ionization measurements must rather be justified by their relative simplicity of interpretation, and the internal consistency of their results. This paper describes one such technique, employing a transverse microwave probe developed to study ionization kinetics in shock-tube flows, ${ }^{1}$ which has performed well by these standards.

The choice of technique for ionization density measurements is usually a process of elimination, based on the particular properties of the medium of interest. In our problem, as in many gas-dynamic situations, we deal with a mildly ionized gas $\left(<10^{-5}\right.$ ions/neutral) at a few percent of atmospheric density. Thus, the techniques of spectroscopic line broadening and continuum intensity measurements, ${ }^{2}$ as well as optical interferometry, ${ }^{3}$ which require relatively high free-charge densities $\left(>10^{16} \mathrm{~cm}^{-3}\right)$ are inapplicable. The magnetic-induction devices ${ }^{4}$ require presumption of the spatial variations in ionization density, which frequently, as in our case, are the principal interest of the experiment. The Langmuir probe, ${ }^{5}$ and the related "double probe," seem technically the simplest devices, but their interpretation is complicated by the aerodynamic, thermal, and electrical disturbances they introduce into the flow under study. The microwave probe techniques must use a frequency closely matched to the electron plasma frequency of the medium under study, and the corresponding wavelength then limits the spatial resolution of the measurement. Fortunately, in our problem, as in many aerodynamically generated ionized gas regions, the axial gradients are not severe, and hence the available resolution

\footnotetext{
${ }^{1}$ R. G. Jahn and K. H. Harwell, "Initial Ionization of Shock-Heated Argon" (to be published).

${ }_{2}$ H. E. Petschek, P. H. Rose, H. S. Glick, A. Kane, and A. Kantrowitz, J. Appl. Phys. 26, 83 (1955).

${ }^{3}$ R. A. Alpher and D. R. White, Phys. Fluids 2, 162 (1959).

${ }^{4}$ S. C. Lin, E. L. Resler, A. R. Kantrowitz, J. Appl. Phys. 26, 95 (1955).

I. Langmuir and H. M. Mott-Smith, Gen. Elec. Rev. 27, 449, 538, 762, $810(1924)$.

1,270 (1957).
}

is adequate. On this basis, the microwave techniques emerge as the least cumbersome of the possibilities.

Of the several schemes for microwave interaction with an ionized gas, the broad distinction is between those involving bounded propagation, as in a wave guide or resonant cavity, and those permitting free propagation of the wave train through the medium of interest. The cavity techniques have so far been more widely used in laboratory studies of electrically ionized gases, ${ }^{7,8}$ principally because of the better definition of the boundary conditions and modal patterns of the fields in the regions of interest. The free-propagation schemes are less closely tied to prescribed geometries, however, and hence promise greater versatility of application to shock tubes, tunnels, and other conventional gas-dynamic facilities. The propagation may be parallel or transverse to the flow, depending on the orientation of the ionization gradients of interest. In practice, the transverse probe is simpler to construct and interpret since the transmitting and receiving antennas are clearly removed from the flow. The distinction between free and bounded propagation is, of course, somewhat artificial. The finite size of the gas body of interest must inevitably introduce certain boundaries into any propagation geometry. Indeed, if the depth of gas probed by a "free" beam is small enough, it may be more reasonable to approach the problem from the standpoint of the interaction of a guided wave with a terminal impedance. ${ }^{9,10}$ In the following, however, we restrict ourselves to the unbounded transverse propagation of a microwave beam through a body of ionized gas at least a few wavelengths in extent in all three dimensions.

\section{THEORETICAL BASIS}

The theory of the interaction of electromagnetic waves with an ionized gas has been considered extensively at many levels of elegance. ${ }^{11-13}$ Here we outline only a very simple approach, felt to be adequate for the interpretation of the experimental data returned by a transverse probe. The usual

\footnotetext{
7 M. A. Biondi and S. C. Brown, Phys. Rev. 76, 1697 (1949).

8 K. B. Persson Phys. Rev. 106, 191 (1957).

9 S. C. Iin, R. A. Neal, and W. I. Fyfe, Avco-Everett Research Rept. 105 (1960). S. C. Lin and B. Kivel, Phys. Rev. 114, 1026 (1959).

${ }_{10}$ F. L. Tevelow, and H. D. Curchack, Diamond Ordnance Fuze Laboratories, TR-962 (1961).

11 R. G. Jahn, Guggenheim Jet Propulsion Center, California Institute of Technology, TN-2 (1960).

${ }_{12} \mathrm{~S}$. Chapman and T. G. Cowling, The Mathematical Theory of Non-Uniform Gases (Cambridge University Press,
New York, 1952), Chap. 18 .

${ }_{13}$ H. Margenau, Phys. Rev. 69, 508 (1946); 109, 6 (1958).
} 
manipulation of Maxwell's equations yields a harmonic wave equation for a uniform medium of finite conductivity but net charge neutrality, of the form

$$
\nabla^{2} \mathbf{E}+k_{0}^{2}[1-i(\sigma / \mathbf{\epsilon})] \mathbf{E}=0,
$$

where $\omega$ is the impressed angular frequency, $k_{0}=$ $2 \pi / \lambda_{0}=\omega / c_{0}$ the vacuum propagation exponent, $i$ the imaginary unit, $\epsilon$ the dielectric permittivity, and $\sigma$ the electrical conductivity. The plane-wave solutions are transverse, in the form

$$
\mathbf{E}=\mathbf{E}_{0} \exp \left[i\left(\omega t \mp k^{*} z\right)\right],
$$

where

$$
k^{*}=k_{0}[1-i(\sigma / \omega \omega)]^{\frac{1}{2}}
$$

is a complex propagation exponent, indicative of the wavelength and the attenuation of the wave motion in the conducting medium.

In applying this formulation to propagation in an ionized gas, the somewhat academic choice must be made whether to regard the oscillations of the free electrons induced by the imposed field as a polarization current, hence contributing to the "dielectric" permittivity $\epsilon$, or as a real current, constituting a conductivity of the medium. The ultimate results for any practical problem are the same, and the latter point of view has been adopted arbitrarily. The development then proceeds from the identification of an "ensemble-average" electron, whose drift velocity defines the current per particle, and satisfies the heuristic equation of motion,

$$
m \dot{\mathrm{v}}_{d}+m \nu_{c} \nabla_{d}=e \mathrm{E} e^{i \omega t} .
$$

This relation may alternatively be regarded as a definition of the quantity $\nu_{c}$, an effective collision frequency for momentum transfer of electrons on heavy particles. The explicit evaluation of $\nu_{c}$ from atomic cross sections is discussed in several standard references, ${ }^{14}$ and elsewhere.$^{15}$ It can be estimated from the single-particle momentum-transfer (diffusion) cross sections $Q_{d}$ averaged over the appropriate electron distribution function:

$$
\nu_{c} \approx \sum_{i} N_{i} \overline{Q_{u_{j}} v}
$$

where $N_{i}$ is the number density of the $j$ th species of heavy particles (molecules or ions). Actually, as will be shown, the effective $\nu_{c}$ may be evaluated

${ }^{14} \mathrm{H}$. S. W. Massey and E. H. S. Burhop, in Electronic and Ionic Impact Phenomena (Oxford University Press, New York, 1952), Chap. 1. S. Chapman and T. G. Cowling, ibid.

${ }_{15}$ D. L. Schultz, Colston Papers, Vol. XI (Butterworths Scientific Publications, London, 1959), p. $301 \mathrm{ff}$. from two simultaneous microwave measurements on the gas of interest, hence need not be known precisely in advance.

Solution of (4) yields a complex conductivity for the ionized gas:

$$
\sigma^{*}=\frac{n e v_{d}}{|\mathbf{E}|}=\frac{n e^{2}}{m\left(\nu_{c}+i \omega\right)}=\epsilon\left(\frac{\omega_{p}^{2}}{\nu_{c}+i \omega}\right),
$$

where $n$ is the free-electron number density, and

$$
\omega_{p}=\left(n e^{2} / \epsilon m\right)^{\frac{1}{t}}
$$

is the familiar electron plasma frequency, i.e., the frequency of natural oscillations of the free-electron component. (Conversely, for a given angular field frequency $\omega$, we may define a resonant electron density, $\left.n_{p}=\epsilon m \omega^{2} / e^{2}\right)$.

The real and imaginary parts of the propagation exponent then follow from substitution of (6) into (3):

$$
\begin{aligned}
& k^{*}=k_{r}-i k_{i} \\
& k_{r}=\frac{k_{0}}{\sqrt{2}}\{(1-P) \\
& \left.\quad+\left[(1-P)^{2}+P^{2}\left(\frac{\nu_{c}}{\omega}\right)^{2}\right]^{\frac{1}{3}}\right\} \\
& k_{i}=\frac{k_{0}}{\sqrt{2}}\{-(1-P) \\
& \left.+\left[(1-P)^{2}+P^{2}\left(\frac{\nu_{c}}{\omega}\right)^{2}\right]^{\frac{1}{3}}\right\}
\end{aligned}
$$

where

$$
P=\frac{\left(\omega_{\nu} / \omega\right)^{2}}{1+\left(\nu_{c} / \omega\right)^{2}}
$$

Thus, the two functions $k_{r}\left(n, v_{c}\right)$ and $k_{i}\left(n, v_{s}\right)$ completely characterize plane-wave propagation in an ionized gas of free-electron density $n$, and effective collision frequency $\nu_{c}$.

In any practical laboratory experiment, the ionized gas under study is confined to a certain finite region, and a probing mierowave train must enter and leave the specimen through interfaces or transition zones, on its way from transmitter to detector. The response of such a probe must then be formulated in terms of the appropriate boundary-value problem. The simplest geometry with hope of experimental realization is that of a plane wave incident normally on a uniform plane slab of ionized gas (see Fig. 1). Allowing both positive- and negative-going waves of form (2) inside of the slab, and requiring continuity of both the electric and magnetic components 


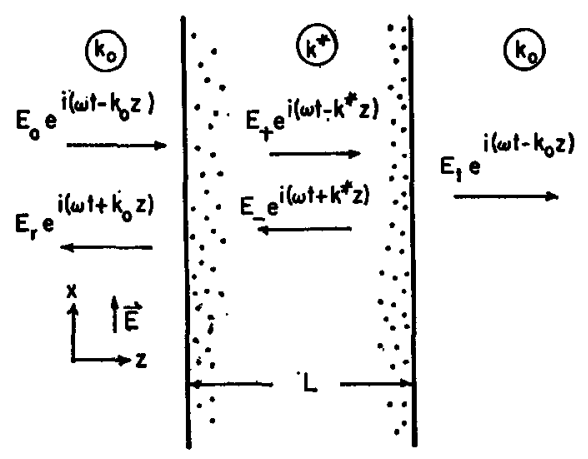

FIG. 1. Plane wave-plane slab interaction geometry.

of the total wave fields at the two interfaces yields expressions for the complex reflection and transmission coefficients ${ }^{16}$ :

$$
\begin{aligned}
\frac{E_{r}}{E_{0}}= & R e^{i \phi_{r}} \\
& =\frac{\left(k_{0}^{2}-k^{* 2}\right)\left(e^{i k^{*} L}-e^{-i k^{*} L}\right)}{\left(k_{0}+k^{*}\right)^{2} e^{i k^{*} L}-\left(k_{0}-k^{*}\right)^{2} e^{-i k^{* L}}},
\end{aligned}
$$

$$
\begin{aligned}
\frac{E_{t}}{E_{0}}= & T e^{i \phi_{t}} \\
& =\frac{4 k_{0} k^{*}}{\left(k_{0}+k^{*}\right)^{2} e^{i k^{*} L}-\left(k_{0}-k^{*}\right)^{2} e^{-i k^{*} L}},
\end{aligned}
$$

where $R, T, \phi_{r}$ and $\phi_{l}$ are real scalars and the notation is that of Fig. 1. These relations define four potentially measurable quantities, the amplitudes and relative phases of the waves reflected from and transmitted through the slab, each of which is a separate function of $n$ and $\nu_{c}$. At microwave frequencies, the available detection devices essentially

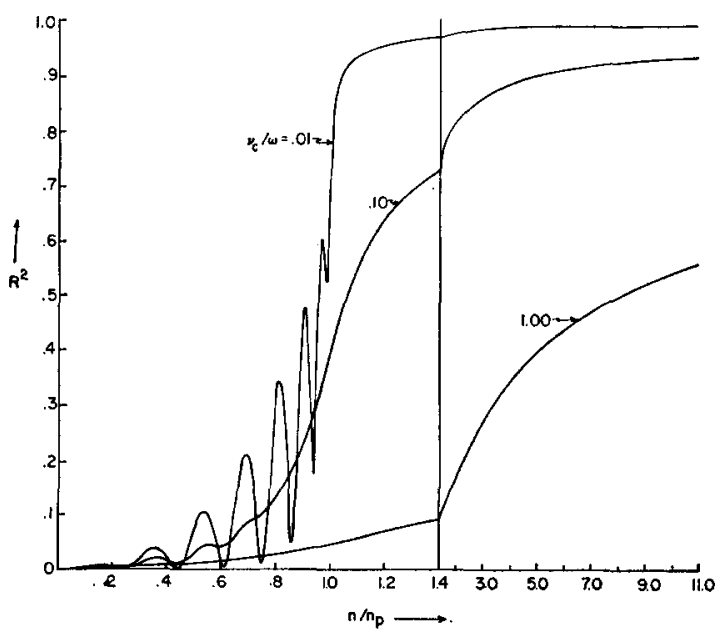

Fia. 2. Reflection from a plane slab of ionized gas, $L=4 \lambda_{0}$.

18 J. A. Stratton, Electromagnetic Theory (Mo(rraw-Hill Book Company, Inc., New York, 1941), Chap. IX.

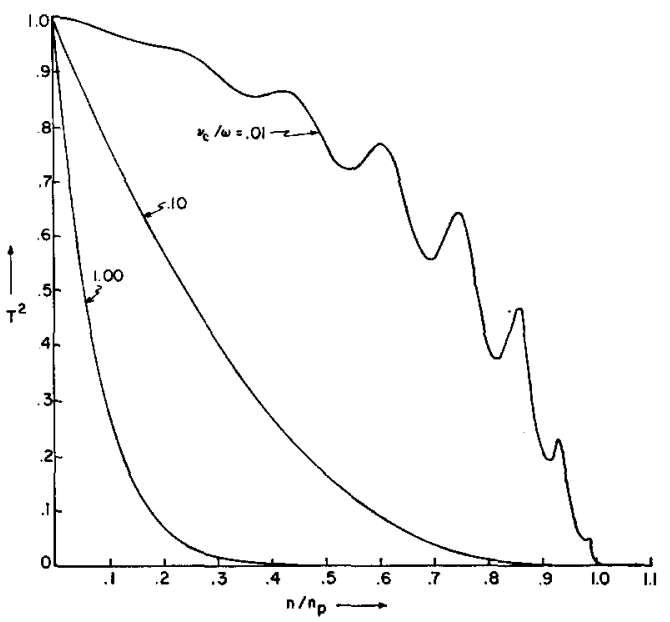

Frg. 3. Transmission through a plane slab of ionized gas, $L=4 \lambda_{0}$.

measure power, e.g., $R^{2}$ and $T^{2}$. The phases are measurable only by an interference of the reflected or transmitted signal with an undisturbed component, for example:

$$
\begin{aligned}
& P_{r}=1+R^{2}-2 R \cos \phi_{r}, \\
& P_{t}=1+T^{2}-2 T \cos \phi_{t} .
\end{aligned}
$$

Typical solutions of the relations (10), (11), (8), (9) for the measureables $R^{2}, T^{2}, P_{r}, P_{t}$, obtained on an IBM 7090 computer, ${ }^{17}$ are shown in Figs. 2-5. In the cases shown it may be seen that for very small ratios of collision frequency to field frequency $\nu_{c} / \omega$ nearly periodic fluctuations in $R^{2}$ and $T^{2}$ occur as the free-electron density increases from zero. These arise from the interference of the primary and

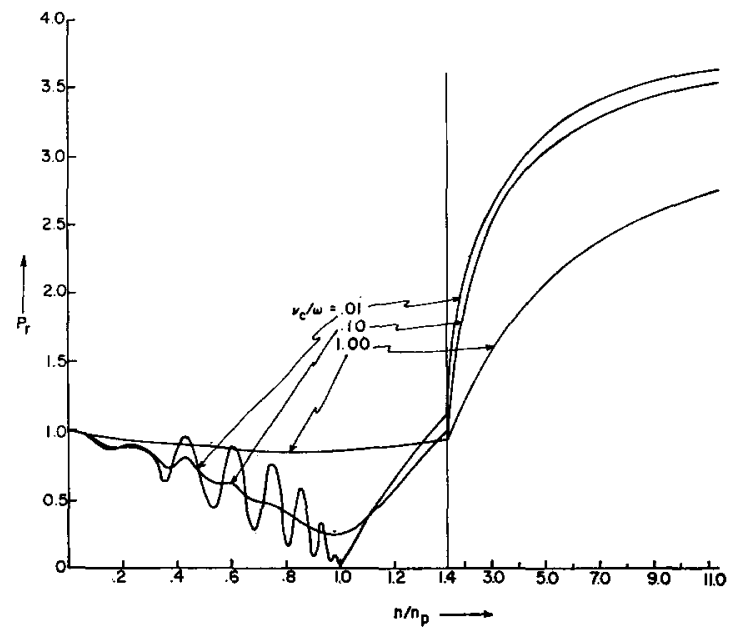

Fig. 4. Reflected phase measureable, $P_{r}=1+R^{2}-2 R \cos \phi_{r}$, for a plane slab of ionized gas, $L=4 \lambda_{0}$.

${ }^{17}$ We are indebted to the Western Data Processing Center at UCLA. 


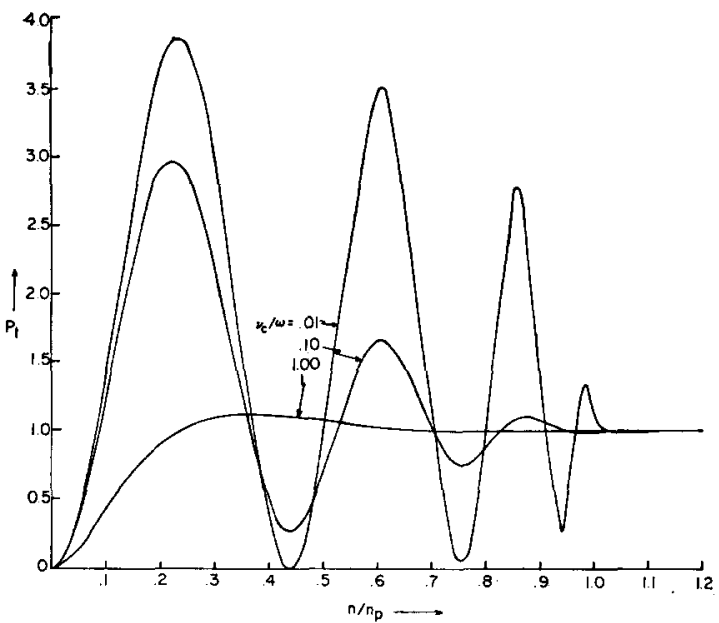

FIG. 5. Transmitted phase measureable, $P_{t}=1+T^{2}$ $-2 T \cos \phi_{t}$, for a plane slab of ionized gas, $L=4 \lambda_{0}$.

multipass signals, as in a conventional parallel-plate interferometer. As the resonant density $n_{p}$ is approached, the attenuation increases, discriminating against the components which must make two additional passes through the slab, and the interference disappears. These interference bumps also become less pronounced for the larger collision frequencies where serious damping sets in before the surface reflections become significant. The fluctuations in the phase measureables $P_{r}$ and $P_{t}$ are seen to be similarly depressed by the larger collision frequencies.

From a more extensive set of calculations, $R^{2}$, $T^{2}, P_{r}, P_{t}$ are found in general to cover somewhat different ranges of sensitivity to $n$, depending on the

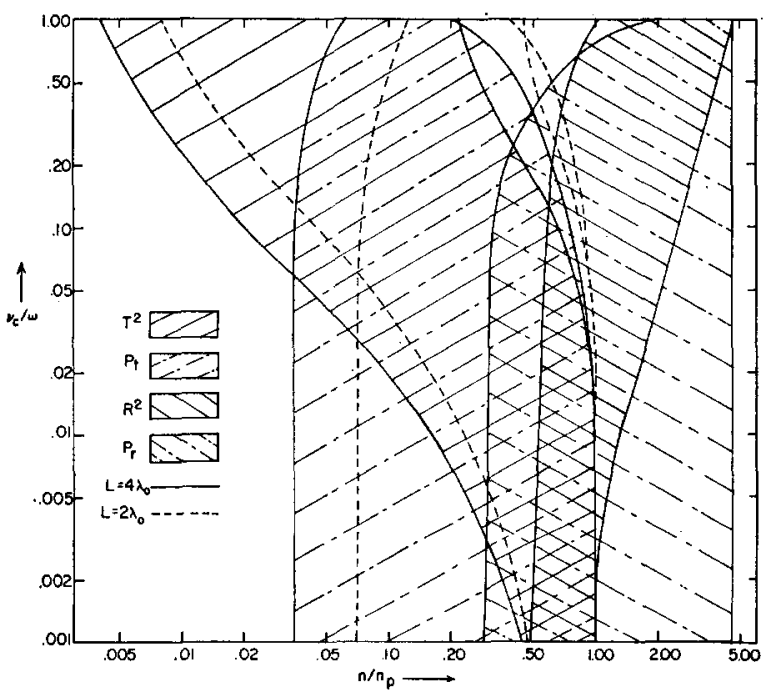

Fra. 6. Domains of electron density and collision frequency accessible to the four microwave measureables, $R^{2}, T^{2}, P, P$ Solid lines, $L=4 \lambda_{0}$; dotted lines, $L=2 \lambda_{0}$. relative collision frequency $\nu_{c} / \omega$, and the relative slab width $k_{0} L$. Figure 6 displays a schematic map of the ranges of $n / n_{p}$ accessible to the four types of measurement for a slab width $L=4 \lambda_{0}$, using the following arbitrary criteria of measureability:

$$
\begin{array}{r}
\left|M-M_{0}\right| \geq 0.05 \\
\left|M-M_{1}\right| \geq 0.05 \\
\frac{d M}{d\left(n / n_{\nu}\right)} \geq 0.05
\end{array} \quad M=\left\{\begin{array}{l}
R^{2} \\
T^{\prime \prime} \\
P_{r} / 4 \\
P_{t} / 4
\end{array},\right.
$$

where $M_{0}$ is the asymptotic value of the measureable for $n \rightarrow 0 ; M_{1}$ the asymptotic value for $n \rightarrow \infty$.

The transmitted amplitude is seen to have its range of sensitivity just below the resonant electron density; the reflected amplitude range straddles $n_{p}$. For the lower collision frequencies, the phase measurements substantially extend the accessible range of electron densities above and below that determinable by the amplitude measurements. Throughout most of the plane, the ranges of at least two of the quantities overlap, indicating that their simultaneous measurement would provide an internal check or, alternatively, permit evaluation of $\nu_{c}$ as well as $n$. In one narrow belt in the plane, all four quantities should be simultaneously measureable; directly above it lies a regime inaccessible to any of them.

A change in the relative slab width primarily alters the range of $T^{2}$ and $P_{t}$, since $R^{2}$ and $P_{r}$ are dominated by the first surface contribution. The dotted lines in Fig. 6 outline the $T^{2}$ and $P_{t}$ zones for a slab of one-half the thickness $\left(L=2 \lambda_{0}\right)$.

\section{DESIGN OF THE PLANE PROBE}

Simply stated, the microwave probe is designed to simulate as closely as possible the plane waveplane slab geometry evaluated above. Clearly, this first requires that the ionized gas under study be provided in a rectangular, dielectric test section of some sort, across which the ionization properties are essentially uniform. Although this is far from a trivial requirement, it can be established adequately in certain gas-dynamic facilities. In the following, it will be presumed that this two-dimensional uniformity prevails. Modifications to the plane slab theory for certain typical inhomogeneities are discussed briefly later, and in detail in other papers. $^{18}$

${ }^{18}$ F. A. Albini and R. G. Jahn, J. Appl. Phys. 32, 75 (1961), and Guggenheim Jet Propulsion Center, California Institute of Technology, TN-3. 
Given this gas uniformity, it remains to establish a plane transverse field propagating normally to the slab, with a frequency commensurate with the ionization density of interest. In some applications these requirements may be mutually exclusive, since the latter prescribes a particular wavelength, which must however be significantly smaller than the $x$ dimension of the test section if the former is to be satisfied. Conversely, these requirements establish a lower limit to the electron density which can be probed in this way. In our application, for example, the shock-tube test section has a 5cm-square cross section. The maximum standard wavelength which can be expected to maintain a satisfactory plane front parallel to this dimension is the $1.25-\mathrm{cm} K$ band, which thus dictates a range of sensitivity centered on a resonant electron density of about $7 \times 10^{12} \mathrm{~cm}^{-3}$.

The radiation and detection of the probing field is best achieved by a pair of identical pyramidal horns, looking at each other across the test section, and suitably matched to the walls of that section. The planarity of the radiated wave front is determined by the apex angles of the horn. The amount of longitudinal component in the wave train's magnetic vector depends on the ratio of the width of the horn mouth in the $\mathbf{H}$ plane, to the wavelength. ${ }^{19}$ The focusing and collimation of the radiated field in the near and intermediate field regions where one must normally operate is a complex function of both horn-mouth dimensions. ${ }^{20}$ The horn dimensions selected must thus be a compromise which best optimizes the field pattern for the particular application of interest. In our case, since the ionization patterns are essentially one-dimensional in the flow direction, there is the additional need to minimize the effective extent of the field in the direction parallel to the gas flow, while maintaining good field uniformity in the transverse directions, i.e., a "fan" pattern. A series of graphical studies, some empirical data from earlier experiments, and certain near-field antenna calculations ${ }^{20}$ were invoked to yield the horn dimensions shown in Fig. 7 . The wave fronts radiated by such horns are calculated to be plane to $1 / 16$ of a wavelength. When assembled on the test section, the focus of each horn was to fall nearly on the aperture of the other. Subsequent tests on the field patterns set up with both horns in place on the test section indicated

${ }^{19}$ F. E. Borgnis and C. H. Papas, in Handbuch der Physik, edited by $S$. Flügge (Springer-Verlag, Berlin, 1958), Vol. XVI, p. $348 \mathrm{ff}$.

${ }_{20}$ F. B. Harris, Jr., Rome Air Development Center TN-58-252 (1958).

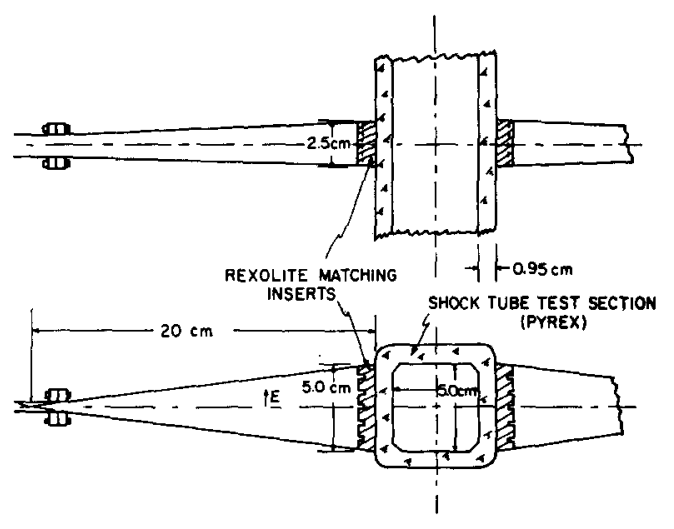

Fig. 7. Microwave horn assembly on test section.

that $90 \%$ of the field energy received by the detecting horn passed within a belt extending one wavelength beyond the geometrical aperture of the horns along the axis of the test section, which essentially confirmed the predictions of the earlier graphical analysis. Very little signal could be detected outside of the test section in the $\mathbf{E}$ plane, indicating that the collimation in that dimension was good.

The walls of the test section should be dielectric for at least a few wavelengths from the horn position to preclude the stray fields from setting up a guided wave propagating in the axial direction in the gas flow channel. Ideally, this dielectric should be nearly lossless to conserve the signal, but for most purposes slightly lossy Pyrex or quartz will suffice. Because all such materials have rather high dielectric permittivities, the four interfaces between source and detector contribute large reflections which must either be included in the data interpretation via a more complex boundary value analysis, or physically compensated by some means, i.e., "matched." The most direct matching procedure would be to make the walls precise half-wave plates by gradually reducing their thickness by grinding until zero reflection is observed. Where this is impractical, as it was in our case, the same effect can be achieved by adding to the outer surfaces of the walls pieces of dielectric material tailored to make the combination of wall plus matching element reflectionless. The tailoring method actually used was to cut a series of rectangular slots across the face of the matching pieces in the direction perpendicular to the E vector (see Fig. 7). The reflection coefficient of the composite set of Pyrex walls and matching pieces was then measured as a function of the width and depth of these slots until, largely by trial and error, a minimum was obtained. In this way it was possible to reduce the net reflection of the entire 


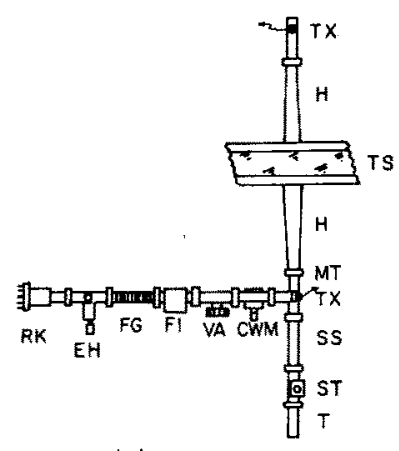

(a)

(b)

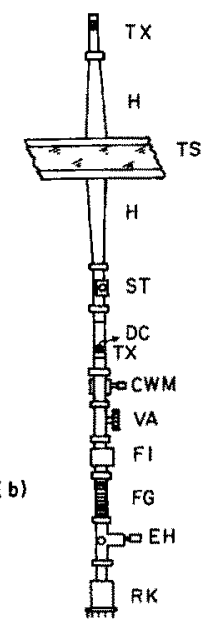

Fra. 8. Two microwave circuits for ionized gas diagnostics: (a) reflection detector on magic tee; (b) reflection detector on directional coupler. Notation: RK, reflex klystron; EH, E, and $H$ plane tuner; FG, section of flexible waveguide; $F I$, ferrite isolator; VA, variable attenuator; CWM, cavity wave meter (absorption type); T, termination; ST, stub tuner; SS, straight section; TX, tunable crystal mount; $\mathrm{H}$, horn; MT, magic tee; DC directional coupler; TS, test section (dielectric).

assembly to about $1 \% .^{21}$ It is shown in another paper $^{11}$ that the reduction of the container walls to effective half-wave plates by either method reduces the problem to the simple slab case outlined earlier, i.e., a slab of ionized gas bounded by air on each side, with no further reference to the intervening walls.

The microwave circuitry used to drive the field horns may be quite straightforward, depending in detail on the particular measurements to be made. Two circuits used in the present experiments are shown in Fig. 8. The transmitted amplitude is measured by a crystal detector attached to the receiving horn. The reflected amplitude is most directly measured by a detector on a directional coupler, as shown in the first circuit. It may likewise be measured via a matched magic tee in the balanced arm circuit also shown in Fig. 8. In this case the crystal signal can be made sensitive to the phase of the reflection, which may or may not be desired. In general, the phases of the reflected and transmitted signals are determined by some form of interferometry, for which many standard circuits are available. ${ }^{22}$ In our case, little use has been made of the reflected phase, since the main interest is in the lower electron densities. The trans-

11 The construction and matching of the horns was performed by the DeMornay-Bonardi Corporation, Pasadena, under the supervision of Dr. G. Fonda-Bonardi and the author.

${ }_{22}$ C. B. Wharton, University of California Radiation Laboratory (Livermore), No. 4836 (Rev.) (1957). mitted phase is very useful in this range, however, and can be obtained with a simple modification of the directional coupler circuit. Namely, after the circuit has been matched as closely as possible, a very small mismatch is purposely introduced at the transmission termination. A second disturbance is then added by the stub tuner preceding the first horn, of such a size and phase that it just cancels the other, making the ambient signal seen by the reflection detector on the directional coupler zero. Subsequent introduction into the test section of a gas of electron density so low that no significant reflection occurs, produces a phase change in the small signal which returns through the gas from the far mismatch, adequate to unbalance the original null at the reflection detector. Thus, for a linear buildup of electron density, for example, the first response of this detector is a series of periodic maxima and minima, several of which may be clearly observable before the actual reflection from the gas sets in.

\section{INTERPRETATION}

To illustrate the application of the microwave probe to a particular problem, more specific reference will be made to the ionization-kinetics experiment for which it was originally developed. The details of this research are covered elsewhere ${ }^{1}$; for the present purpose the essence of the situation is a monotonic increase in ionization density following the passage of a strong shock wave through argon in a glass-walled shock-tube test section. The shock wave imparts random thermal energy to the argon atoms, raising the (translational) temperature of the gas to several thousand ${ }^{\circ} \mathbf{K}$. Subsequently, via various particle collisions, the ionization density builds up monotonically to its equilibrium value. During the course of this buildup, the free-electron density passes through the range accessible to the 1.25 -cm microwaves. Specifically, the experiment consists of monitoring the reflection, transmission and phase shifts of the microwave beam as functions of time after the passage of the shock wave, using either of the two circuits of Fig. 8.

A tracing of a typical dual-beam oscilloscope record of the microwave responses are shown in Fig. 9. The chopped upper beam records $R^{2}$ and $T^{2}$; the lower beam records $R^{2}$ at ten times greater amplification. The reflection is seen first to present the small interference bumps indicative of $\phi_{t}$, generated in the manner described in section III, and then proceeds to its larger reflected amplitude response. From the observation that $R^{2}$ becomes 
significant slightly after $T^{2}$ has reached zero we may immediately estimate that $\nu_{e} / \omega \approx 0.1$ for the gas under study (Fig. 6). A more precise determination of $\nu_{c} / \omega$ emerges from the detailed reduction of the transmission and reflection profiles by the following method (Fig. 10). Since $\phi_{t}$ is relatively insensitive to $v_{c} / \omega$, a graph of $n$ vs $t$ is begun with points calculated from the observed positions of the little maxima and minima in the early portion of the reflection trace, using the estimated $\nu_{c} / \omega$. The $R^{2}$ response is then reduced for several values of $\nu_{\mathrm{c}} / \omega$ surrounding the estimated value. These curves are added to the plot, and found to emerge from a common value of $n$ at the lowest detectable $R^{2}$. Finally, the $T^{2}$ response is also reduced for these values of $\nu_{c} / \omega$, and that curve selected which best overlaps the $\phi_{t}$ bump points for low $n$ and extrapolates through the corresponding $R^{2}$ curve for the higher $n$. This fitting is found to be quite unambiguous. Choice of a slightly higher or lower $\nu_{c} / \omega$ would yield a $T^{2}$ eurve deviating noticeably from the end points and slopes established by the $\phi_{t}$ and $R^{2}$ curves for the same $\nu_{c} / \omega$.

\section{DATA CORRECTION}

The process for evaluating $n(t)$ and $\nu_{c}$ outlined above must be preceded by some reconciliation of the departures of the experiment from the plane slab idealizations. For example, the crystal diodes used as transducers of the microwave energy respond only approximately to the square of the local field amplitude, and should be calibrated in place at the signal level, carrier frequency, and response frequency typical of the actual data. More troublesome are the corrections for the deviations of the probing field from a plane wave, and the deviations

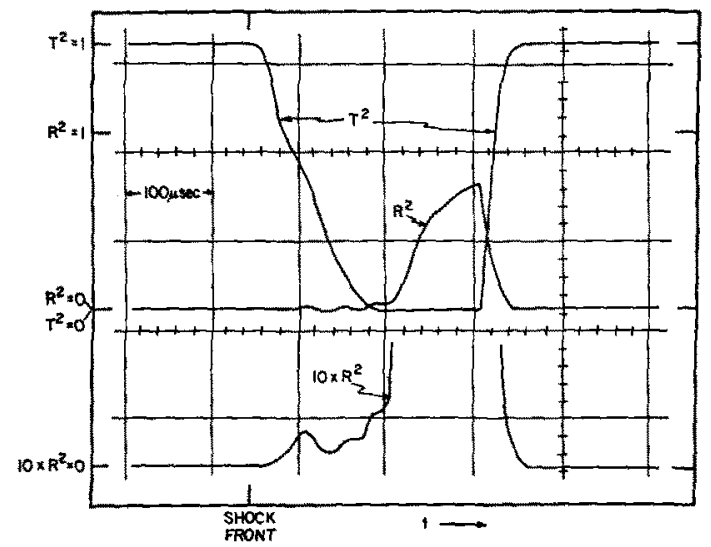

FrG. 9. Response of transmission and reflection detectors to passage of Mach 9 shock wave in argon (initial pressure $5 \mathrm{~mm}$ ).

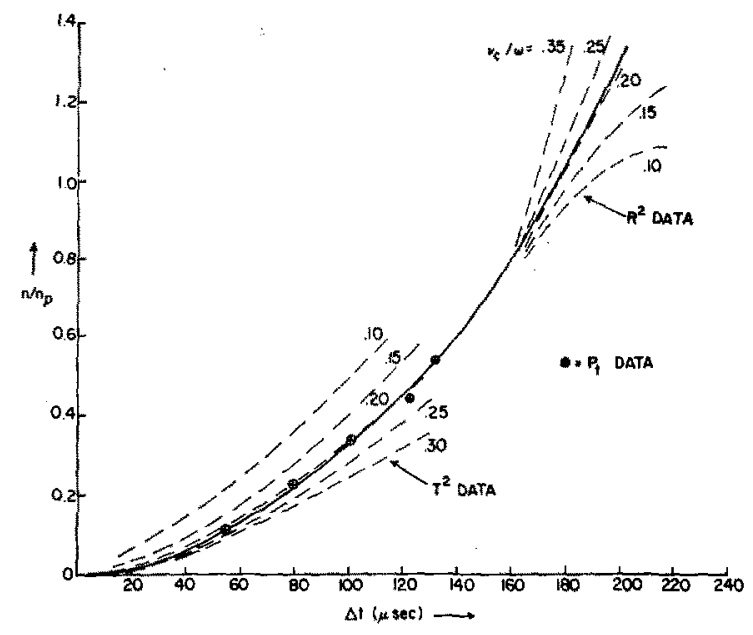

Frg. 10. Construction of composite $n(t)$ curve by identification of effective collision frequency (see text).

of the ionization profiles from the plane slab. Neglecting the latter for the moment, the main complication introduced by a curved wave front is its vulnerability to refraction. In other words, the observed decrease in the transmitted signal may arise from two causes: (1) the bulk attenuation and interface reflections accounted for in the slab theory, and (2) distortion of the radiation patterns of the two horns by the introduction of the gas of lower index of refraction between them. The latter effect could not occur in a truly one-dimensional geometry, but must affect the fringing fields of finite aperture horns. Lacking any permanent media of refractive index less than one with which to calibrate the probe against this effect, recourse was made to the theoretical calculations of near-field horn patterns ${ }^{20}$ which indicate that the field intensities on the horn axis in this region scale as the product of wavelength and axial distance from the horn mouth. A series of calibrations were then performed in which the received signals were studied as a function of horn separation, and from this and the relations between $\lambda$ and $T^{2}$ implicit in the slab calculations, the refraction losses were estimated. The correction involved varied from zero at $T^{2}=0$ and 1 , to about $5 \%$ of total transmission at an intermediate value, small enough to justify the somewhat cavalier treatment of it in this situation. For less favorable geometries, or less well collimated horn fields, more detailed calibration against this effect could be required.

The sensitivity of the probe to departures from the assumed plane slab ionization profile, i.e., its response to ionization gradients in the direction of propagation, is clearly a crucial point. The obvious 
concern is that the gas-dynamic boundary layers, and/or the electrical boundary effects will replace the assumed discrete interfaces by smooth transition zones of sufficient breadth to produce first-order deviations from the plane slab solutions. This problem has been studied in detail and extensive results are presented elsewhere. ${ }^{18}$ Briefly, it is found that the solutions for slabs bounded by transition zones in excess of about $0.2 \lambda_{0}$ differ significantly from those bounded by sharp interfaces in all of the measureable quantities. If the total width of the gas sample is at least a few wavelengths, $R^{2}$ is more seriously affected than $T^{2}$, since the latter responds mainly to the integrated path properties, the former to the first surface. As this surface becomes more diffuse, $R^{2}$ decreases, and $T^{2}$ increases. Specifically, for the dimensions and parameters used above, $R^{2}$ would be some $20 \%$ lower, $T^{2}$ about $5 \%$ higher in their midranges if transition zones of $\frac{1}{4} \lambda_{0}$ replaced the sharp interfaces. The phase measureables are also sensitive to protraction of the boundary regions since the effective path lengths inside and outside of the gas sample are altered thereby. In the above case, $\phi_{R}$ would decrease by some $90^{\circ}, \phi_{T}$ by about $60^{\circ}$ for the $\frac{1}{4} \lambda_{0}$ transitions.

The dependence of the measureables on the character of the boundary regions clearly can lead to misinterpretation of the free-stream electron density and collision frequency. Unfortunately, the estimation of the transition-zone width is seldom straightforward; calculation of its detail profile is usually impossible. The gas-dynamic boundarylayer profile is relevant only if local ionization equilibrium prevails through it. The electron-density profile accompanying the ambipolar diffusion to the test section wall is more appropriate, but difficult to evaluate because of uncertain atomic cross sections in the regions of temperature gradient, and unknown electrical boundary conditions at the wall surface. In our current experiments, rudimentary boundary layer $^{23}$ and ambipolar diffusion ${ }^{24}$ calculations in-

${ }^{23}$ H. Mirels, NACA TN 3401 (1955); NACA TN 3278 (1956); NACA TN 3712 (1956).

${ }_{24}$ S. C. Brown, Basic Data of Plasma Physics (John Wiley \& Sons, Inc., New York, 1959), Chap. 3. dicate upper limits for transition-zone widths large enough to alter the $R^{2}$ and $P_{r}$, signals significantly. The manifestation of such an effect would be a drooping of the $n$-vs- $t$ curves deduced from these measureables below the composite curve at the later times in the flow behind the shock, when the transition zones had grown large (see Fig. 10). Some such behavior is indeed seen, but never enough to justify alteration of the composite curve. Attention has been called to this complication, however, since (1) steady flows of ionized gases, and less transient unsteady flows, will tend to generate much broader boundary regions; (2) the use of higher probing frequencies, attractive from the standpoint of spatial resolution, will tend to aggravate this difficulty.

\section{SUMMARY}

The transverse microwave probe described above permits immediate qualitative study of a specific range of ionization densities and collision frequencies in nearly one-dimensional gas flows. In this range, quantitative information of good precision can be extracted from detailed comparison of the amplitudes and phases of the reflected and transmitted signals, if the geometry of the gas flow and probing fields correspond closely to the plane slab model, or if adequate compensations for deviations can be made. The choice of frequency and the design of the antennas for a given probe are compromises to the various conditions of sample size, free-electron density, and the extent and severity of the axial and transverse variations in the electron density in the gas under study, as detailed above.

\section{ACKNOWLEDGMENT}

The author is greatly indebted to Dr. G. FondaBonardi for his recommendations on the design of the microwave horns and the associated circuitry, and for several helpful suggestions on the conduct of the experiments. K. Harwell performed much of the technical operation and assisted considerably with data reduction.

This work was supported in part by the U.S. Air Force Office of Scientific Research. 